\title{
SARS-CoV-2 antibody seroprevalence among firefighters in Orange County, California
}

\author{
Verónica Vieira (1) ${ }^{1,2}$ lan W Tang, ${ }^{1}$ Scott Bartell, ${ }^{1,2}$ Matthew Zahn, ${ }^{3}$ \\ Marion Joseph Fedoruk ${ }^{1,2}$
}

'Department of Environmental and Occupational Health, University of California Irvine Susan and Henry Samueli College of Health Sciences, Irvine, California, USA

${ }^{2}$ University of California Irvine Center for Occupational and Environmental Health, Irvine, California, USA

${ }^{3}$ Orange County Health Care Agency, Santa Ana, California, USA

Correspondence to Dr Verónica Vieira, Department of Environmental and Occupational Health, University of California Irvine College of Health Sciences, Irvine, California, USA; vvieira@uci.edu

Received 8 February 2021 Accepted 3 August 2021 Published Online First 25 August 2021

\section{ABSTRACT \\ Objectives We conducted serological SARS-CoV-2 antibody testing from October to November 2020 to estimate the SARS-CoV-2 seroprevalence among firefighters/paramedics in Orange County (OC), \\ California.}

Methods OC firefighters employed at the time of the surveillance activity were invited to participate in a voluntary survey that collected demographic, occupational and previous COVID-19 testing data, and a SARS-CoV-2 immunoglobulin (Ig)G antibody blood test. We collected venous blood samples using mobile phlebotomy teams that travelled to individual fire stations, in coordination with an annual tuberculosis testing campaign for firefighters employed by OC Fire Authority (OCFA), and independently for firefighters employed by cities. We estimated seroprevalence and assessed several potential predictors of seropositivity. Results The seroprevalence was 5.3\% among 923 OCFA personnel tested, with $92.2 \%$ participating. Among firefighters self-reporting a previous positive COVID-19 antibody or PCR test result, twenty-one (37\%) did not have positive $\lg G$ tests in the current serosurvey. There were no statistically significant differences in demographic characteristics between cases and noncases. Work city was a significant predictor of case status $(p=0.015)$. Seroprevalence $(4.8 \%)$ was similar when aggregated across seven city fire departments (42\%-65\% participation). In total, $1486^{\circ} \mathrm{C}$ fire personnel were tested.

Conclusion Using a strong serosurvey design and large firefighter cohort, we observed a SARS-CoV-2 IgG seroprevalence of $5.3 \%$. The seroprevalence among OC firefighters in October 2020 was lower than the general county population estimated seroprevalence (11.5\%) in August. The difference may be due in part to safety measures taken by $\mathrm{OC}$ fire departments at the start of the pandemic, as well as differences in antibody test methods and/or duration of antibody response.

\section{INTRODUCTION}

Firefighters play a vital role in Orange County (OC) California communities by assisting in emergencies, providing emergency medical treatment, and transporting ill or injured individuals, in addition to performing traditional firefighting duties. ${ }^{1}$ Exposure to COVID-19 through public interactions may affect coworkers in shared firehouse spaces and could lead to resource challenges (ie, staffing and response readiness) due to increased medical leave. ${ }^{2}$ Antibody testing can be a useful tool in

\section{KEY MESSAGES \\ WHAT IS ALREADY KNOWN ABOUT THIS SUB- JECT? \\ $\Rightarrow$ Firefighters/paramedics may have substantial risk of exposure to COVID-19 infection due to their public interactions, emergency assistance, and shared workspaces. Transmission among coworkers could lead to reduced staffing and impaired response-readiness.}

\section{WHAT ARE THE NEW FINDINGS?}

$\Rightarrow$ The seroprevalence of SARS-CoV-2 immunoglobulin (Ig)G was 5.3\% among 923 Orange County Fire Authority personnel tested in October 2020 (92.2\% participation rate), lower than the previously reported seroprevalence $(11.5 \%)$ among residents of Orange County California in August 2020.

$\Rightarrow$ Among 57 firefighter/paramedics who selfreported a previous positive COVID-19 test result, $37 \%$ were no longer positive in the current serosurvey.

HOW MIGHT THIS IMPACT ON POLICY OR CLINICAL PRACTICE IN THE FORESEEABLE FUTURE?

$\Rightarrow$ Although personal protective equipment and other safety protocols likely protected firefighters/paramedics from COVID-19 infection compared with the general county population, prevalence of past COVID-19 infection is likely underestimated by IgG serosurveys given antibody decline over time.

$\Rightarrow$ Clinical significance regarding future immune response is not well understood. Standardised testing methods using more sensitive antibody tests would make comparisons across different populations more meaningful.
(C) Author(s) (or their employer(s)) 2021. No commercial re-use. See rights and permissions. Published by BMJ.

To cite: Vieira $\mathrm{V}$

Tang IW, Bartell S, et al.

Occup Environ Med

2021:78:789-792. understanding seroprevalence within a population by detecting past immune response. ${ }^{3}$ Seroprevalence in other first responder studies conducted from April to July ${ }^{4-8}$ ranged from $1.5 \%$ in Arizona ${ }^{8}$ to $8.9 \%$ in South Florida ${ }^{4}$ among firefighters.

COVID-19 seroprevalence in a sample of the general OC population was estimated at 11.5\% from July to August 2020, ${ }^{9}$ coinciding with southern California's first surge in COVID-19 cases. OC is the sixth most populous county in the country and has the second highest population density among 
California counties. In collaboration with the OC Health Care Agency (OCHCA), the University of California, Irvine (UCI) Center for Occupational and Environmental Health, the OC Fire Authority (OCFA) and city fire departments across the county, we conducted serological SARS-CoV-2 antibody testing from October to November 2020. The objective of this serosurveillance activity was to estimate SARS-CoV-2 seroprevalence among OC firefighters.

\section{METHODS}

\section{Serosurvey design}

This 2-month serosurvey of SARS-Cov-2 IgG antibodies among OC firefighters was part of broader testing efforts supported by OCHCA during the pandemic. Personnel from all 10 fire departments providing emergency medical services in OC were invited to participate. Nine OC cities have their own city fire and paramedic services, with approximately 1000 employees and 61 fire stations. OCFA provides fire and paramedic services for the other 23 cities and all unincorporated areas in OC, with approximately 1300 firefighters and 77 fire stations.

Personnel employed with OCFA or city fire departments during the testing period were invited to participate in a brief questionnaire and COVID-19 antibody testing. OCFA firefighters and staff were administered the questionnaire in-person while city fire department personnel completed it online. The questionnaire included demographic, occupational and residential information, as well as previous COVID-19 testing, diagnosis and symptoms. Participants were encouraged to complete the questionnaire regardless of whether they chose to participate in the antibody test.

Mobile phlebotomy teams travelled to the fire stations to draw venous blood samples. Participants were tested for immunoglobulin (Ig) G antibodies, which informs about previous infection, using the SARS-CoV-2 IgG Reagent Kit in combination with the SARS-CoV-2 IgG Calibrator Kit on the ARCHITECT i system, an FDA approved confirmatory antibody test developed by Abbott Laboratories. The chemiluminescent microparticle immunoassay is designed to detect IgG antibodies to the nucleocapsid protein of SARS-CoV-2. The test is positive for IgG antibodies to SARS$\mathrm{CoV}-2$ if the ratio of chemiluminescent relative light unit (RLU) in the reaction with the sample to the calibrator RLU is $\geq 1.4$. The sensitivity of the test was $99.6 \%$ and the specificity was $100 \% 17$ days post symptom onset. ${ }^{10}$

\section{OCFA cohort}

From October 5 to 23, 2020, OCFA conducted its annual mandatory tuberculosis (TB) testing. Phlebotomists visited multiple fire stations during that time period to draw blood from firefighters for the TB test and, for consenting participants, the SARS-CoV-2 IgG test. UCI staff were also present at the fire stations to invite OCFA firefighters to participate in the voluntary COVID-19 antibody testing and to administer the questionnaire. All firefighters, regardless of whether they participated, provided their employee ID, which allowed linkage to OCFA data (age and race/ethnicity). In addition, non-participants also provided a reason for not participating.

\section{Data analysis}

As our primary analysis, we calculated the seroprevalence of a positive IgG antibody test and descriptive statistics of survey data for OCFA firefighters recruited through the annual TB testing. We used $\chi^{2}$ tests to compare characteristics of cases versus non-cases and participants versus non-participants, excluding participants with missing questionnaire responses for each item. Tests with $\mathrm{p}$ values $<0.05$ were considered statistically significant. Seroprevalence was also calculated for each city. All statistical analyses were performed in R (V.4.0.0, R Foundation).

\section{RESULTS}

A total of 1001 OCFA personnel participated in the 3-week annual TB testing campaign in October and were invited to participate in the surveillance activity. Of those, 896 participated in both the IgG blood test and survey, and 27 participated in just the IgG blood test, for a total of $923(92.2 \%)$ participating in the IgG blood test. Another 26 personnel completed only the questionnaire and 52 declined to participate in either the $\operatorname{IgG}$ blood test or questionnaire, for a total of 78 non-participants of the blood test. Table 1 describes the characteristics of the 1001 OCFA personnel. Those who participated in the IgG blood test $(n=923)$ were further stratified by positive (cases, $n=49)$ and negative (non-cases, $\mathrm{n}=874$ ) $\operatorname{IgG}$ test results. Seroprevalence among this OCFA cohort was 5.3\% (49/923). Among 49 cases who had a previous COVID-19 test, 13 (26.5\%) reported having been asymptomatic. Of the 36 participants who reported a prior positive PCR test, 7 reported being asymptomatic (19.4\%). Twenty-one of the 57 (37\%) personnel self-reporting a previous positive COVID-19 PCR or antibody test did not have a positive IgG test in our serosurvey.

There were no significant differences in demographics between cases and non-cases (table 1). The seroprevalence was $4.1 \%(13 / 318)$ among firefighters who were also paramedics and $14.3 \%(6 / 42)$ among Fire Chiefs, but differences in job position and rank were not statistically significant $(p=0.176)$. Seroprevalence by work city for OCFA fire stations ranged from $1.9 \%$ in Santa Ana to $12.3 \%$ in Garden Grove ( $p<0.001$ for equivalence across all 33 cities). However, case numbers were too small in most cities to provide stable seroprevalence estimates, so the chisquare test may be inaccurate.

Participants and non-participants were similar in age and race (table 1). Non-participants were more likely to be Hispanic than participants but differences were not statistically significant $(26.9 \%$ vs $18.9 \%, p=0.114)$. None of the nonparticipants reported a previous positive COVID-19 test as the reason for not participating. Reasons given were that they were not interested, in a hurry, or had a negative test already. An additional 107 OCFA office staff opted for the voluntary antibody testing and 37 OCFA firefighters were tested after the TB campaign. Less than five were positive among the 144 personnel. Given the difference in the recruitment approach of these personnel compared with the OCFA cohort recruited during the TB testing campaign, we did not include them in our primary analyses. In all, 1067 OCFA personnel participated in the antibody testing.

Firefighters and staff from six of the nine city fire departments participated in the surveillance activity using mobile phlebotomists, with participation ranging from $42 \%$ to $65 \%$, for a total of 415 personnel tested. One fire department opted for testing outside of the fire stations, resulting in only four firefighters participating. Six city fire departments had fewer than five positive cases, so data were aggregated. There were 20 positive cases among 419 total city fire department participants $(4.8 \%)$. There were too few cases to compare characteristics with non-cases in this group. Data from non-participants were not available so we were unable to assess previous COVID-19 results as the reason for not participating. In total, $1486^{\circ} \mathrm{C}$ fire personnel were tested. 
Table 1 Characteristics of Orange County Fire Authority (OCFA) personnel who participated in SARS-CoV-2 lgG blood test ( $\mathrm{n}=923$ ) by case status and comparison with non-participants $(n=78)$

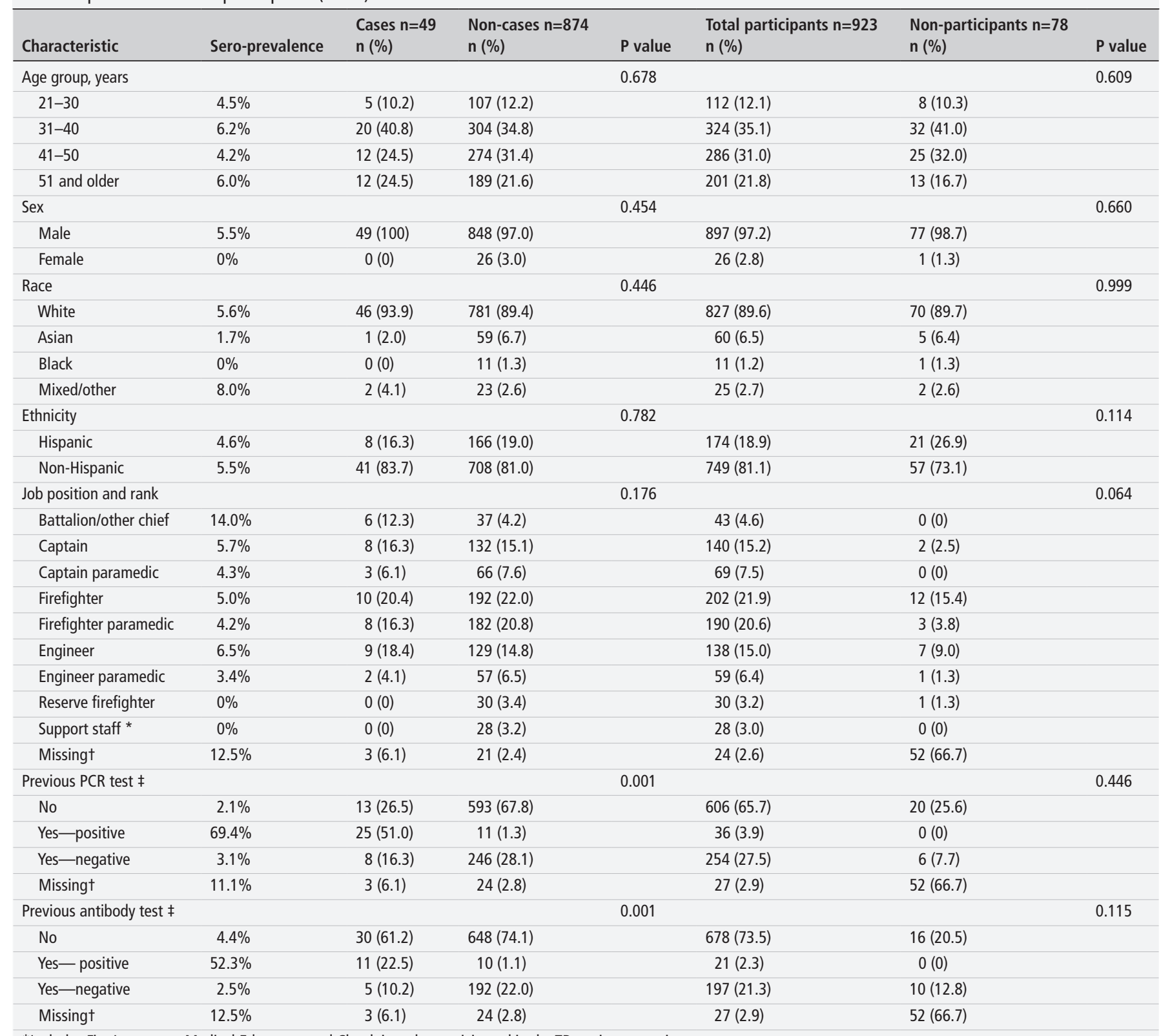

*Includes Fire Inspectors, Medical Educators, and Chaplains who participated in the TB testing campaign.

tMissing values are excluded from the $\chi^{2}$ tests.

\#Firefighters who had a prior positive PCR test and those with a prior positive antibody test were not mutually exclusive.

\section{DISCUSSION}

The seroprevalence of 5.3\% in October 2020 among OCFA firefighters in OC, California is lower than the summer seroprevalence of $11.5 \%$ in the general OC population. ${ }^{9}$ Seroprevalence in this occupational group may be lower due to early changes implemented by fire departments to protect personnel, including daily station disinfection, symptom screening prior to shifts, and encouraging frequent hand washing. In addition, even prior to the pandemic, OCFA required personal protective equipment (PPE) when assisting patients and followed the general guidance from the CDC for emergency medical services. ${ }^{1}$ A study of OC healthcare workers ${ }^{11}$ also attributed considerably lower seroprevalence to PPE usage and hand washing. Differences in antibody test methods ${ }^{9}$ and duration of detectable antibodies post infection may have also contributed to lower seroprevalence. ${ }^{12}$
The 5.3\% seroprevalence among OCFA firefighters was similar to seroprevalence in other densely populated geographic areas of the USA that increased PPE use and took early social distancing measures $(6.7 \%$ among 330 firefighters in Detroit, Michigan $^{5}$; and 4.8\% among 62 firefighters in Washington DC). ${ }^{6}$ In addition, firefighters who were also paramedics were not more likely to test positive for SARS-CoV-2 IgG antibodies compared with non-medic firefighters. Unlike the county-wide serosurvey, ${ }^{9}$ which observed significantly higher seroprevalence among Hispanics, race/ethnicity did not differ among cases and non-cases in the current serosurvey. This may be due to homogeneity of workplace environments inherent among this occupational cohort compared with the general population. Geographically, we also found substantially lower seroprevalence among firefighters than had been reported for the general 
community in certain cities. For example, the seroprevalence was $1.9 \%$ for firefighters working in Santa Ana, but the seroprevalence among residents of that city exceeded $12 \%$ in a community seroprevalence study that was conducted a few months earlier. ${ }^{13}$

A strength of this serosurvey was the high participation rate (92.2\%) among one of the largest firefighter cohorts in the USA. Offering antibody testing synchronously with mandatory annual OCFA TB testing was convenient for participants, enabling a nearly complete sample with minimal selection bias. We also were able to assess differences by firefighter job duties, race/ ethnicity, and work location.

Despite the strengths, we experienced much lower participation rates among the OC city fire departments. Participation in these fire departments was likely biased, as those who had already received a positive COVID-19 PCR test result had less interest in learning their antibody test results. Testing was also limited to $\mathrm{IgG}$ antibodies. Results may have differed if we also tested for IgM and/or other antibodies. ${ }^{4}$

The seroprevalence of SARS-CoV-2 IgG antibodies was relatively low among OC firefighters in October 2020 compared with the general county population. The generally lower seroprevalence of firefighters compared with the general population suggests that PPE measures put into place in March 2020 were effective in protecting OC firefighters. However, prevalence of past COVID-19 infection is likely underestimated by IgG serosurveys given antibody decline over time. Differences in antibody test methods and the duration of an individual's antibody response may also contribute to the lower seroprevalence. Standardised testing methods using more sensitive antibody tests would make comparisons across different populations more meaningful.

Acknowledgements We would like to acknowledge the OCFA, city fire departments of Anaheim, Costa Mesa, Fountain Valley, Fullerton, Laguna Beach, Newport Beach, and Orange, and all the fire department personnel for participating in this surveillance activity. We would also like to thank the numerous serveOC and COEH staff who assisted with this surveillance activity.

Contributors All authors contributed to the research design and manuscript development. VV, SB and IWT completed data collection.

Funding This surveillance activity was supported by the Orange County Healthcare Agency.

Competing interests None declared.

Patient consent for publication Not required.
Ethics approval This study involves human participants but was exempted by our Institutional Review Board. Per federal regulations, public health surveillance activities are deemed not to be research.

Provenance and peer review Not commissioned; externally peer reviewed.

Data availability statement Data are available upon reasonable request. Data from this study are available on request by sending an email message to the corresponding author, Dr Verónica Vieira (vvieira@uci.edu).

This article is made freely available for use in accordance with BMJ's website terms and conditions for the duration of the covid-19 pandemic or until otherwise determined by BMJ. You may use, download and print the article for any lawful, non-commercial purpose (including text and data mining) provided that all copyright notices and trade marks are retained.

\section{ORCID iD}

Verónica Vieira http://orcid.org/0000-0001-7153-4606

\section{REFERENCES}

1 CDC. Coronavirus disease 2019 (COVID-19). cent. Dis. control Prev, 2019. Available: https://www.cdc.gov/coronavirus/2019-ncov/hcp/guidance-for-ems.html

2 Prezant DJ, Zeig-Owens R, Schwartz T, et al. Medical leave associated with COVID-19 among emergency medical system responders and firefighters in New York City. JAMA Netw Open 2020;3:e2016094.

3 Furukawa NW, Brooks JT, Sobel J. Evidence supporting transmission of severe acute respiratory syndrome coronavirus 2 while presymptomatic or asymptomatic. Emerg Infect Dis 2020;26.

4 Caban-Martinez AJ, Schaefer-Solle N, Santiago K, et al. Epidemiology of SARS-CoV-2 antibodies among firefighters/paramedics of a US fire department: a cross-sectional study. Occup Environ Med 2020;77:857-61.

5 Akinbami LJ, Vuong N, Petersen LR, et al. SARS-CoV-2 seroprevalence among healthcare, first response, and public safety personnel, Detroit metropolitan area, Michigan, USA, May-June 2020. Emerg Infect Dis 2020;26:2863-71.

6 Reuben J, Sherman A, Ellison JA. SARS-CoV-2 seroprevalence among first responders in the district of Columbia, may - July 2020. medRxiv 2020.

7 Sabourin KR, Schultz J, Romero J, et al. Risk factors of SARS-CoV-2 antibodies in Arapahoe County first Responders-The COVID-19 Arapahoe serosurveillance study (cases) project. J Occup Environ Med 2021;63:191-8.

8 Shukla V, Lau CSM, Towns M, et al. COVID-19 exposure among first responders in Arizona. J Occup Environ Med 2020;62:981-5.

9 Bruckner TA, Parker DM, Bartell SM, et al. Estimated seroprevalence of SARS-CoV-2 antibodies among adults in orange County, California. Sci Rep 2021;11:3081.

10 Bryan A, Pepper G, Wener MH, et al. Performance characteristics of the Abbott architect SARS-CoV-2 IgG assay and seroprevalence in Boise, Idaho. J Clin Microbiol 2020;58:e00941-20.

11 Brant-Zawadzki M, Fridman D, Robinson PA, et al. SARS-CoV-2 antibody prevalence in health care workers: preliminary report of a single center study. PLOS One 2020;15:e0240006.

12 Self WH, Tenforde MW, Stubblefield WB, et al. Decline in SARS-CoV-2 Antibodies After Mild Infection Among Frontline Health Care Personnel in a Multistate Hospital Network - 12 States, April-August 2020. MMWR Morb Mortal Wkly Rep 2020;69:1762-6.

13 Parker DM, Bruckner TA, Vieira VM. Epidemiology of the early COVID-19 epidemic in orange County, California: comparison of predictors of test positivity, mortality, and seropositivity. medRxiv 2021 